\title{
Asymptomatic Eosinophilic Infiltration with Endoscopic and Histological Findings Consistent with Eosinophilic Gastritis
}

\author{
Kazuki Yamamoto Takeshi Okamoto Katsuyuki Fukuda \\ Department of Gastroenterology, St. Luke's International Hospital, Tokyo, Japan
}

\section{Keywords}

Case report · Endoscopy - Esophagogastroduodenoscopy · Eosinophilic gastroenteritis .

Eosinophilic gastrointestinal disease $\cdot$ Screening

\begin{abstract}
Eosinophilic gastritis often presents with gastrointestinal symptoms in the absence of abnormal endoscopic findings. On the other hand, endoscopic changes due to eosinophilic infiltration in an asymptomatic patient are rare. A 29-year-old woman with a history of asthma on steroid inhalers presented for an annual medical checkup. Esophagogastroduodenoscopy revealed diffuse white granular patches in the body of the stomach, suggestive of eosinophilic gastritis. Histology showed over 100 eosinophils per high-power field, also consistent with eosinophilic gastritis. As the absence of symptoms precluded the diagnosis of eosinophilic gastritis, the patient was diagnosed with asymptomatic eosinophilic infiltration of the stomach.
\end{abstract}

\section{Introduction}

Eosinophilic gastritis (EG) is a rare disorder characterized by eosinophilic infiltration of the gastric wall with symptoms such as abdominal pain, bloating, and nausea. There is a slight male preponderance, with an overall prevalence of $28 / 100,000$ persons in the USA [1]. In Japan, a nationwide survey revealed that $\mathrm{EG}$ is about 5.5 times more prevalent than in the USA and more common than eosinophilic esophagitis [2].

EG presents without abnormal endoscopic findings in over $60 \%$ of cases [3]. On the flipside, endoscopic changes with severe eosinophilic infiltration in an asymptomatic patient 
have not been reported. Herein, we present a case of asymptomatic eosinophilic infiltration of the stomach with endoscopic and histological findings consistent with EG.

\section{Case Report/Case Presentation}

A 29-year-old Japanese woman presented for an annual medical checkup. Her medical history was only significant for bronchial asthma which was well controlled on steroid inhalers. She had received a checkup every year over the past 4 years, and no abnormalities had been noted in laboratory tests or esophagogastroduodenoscopy (EGD).

Laboratory testing was only remarkable for mild eosinophilia of $760 / \mathrm{mm}^{3}$, compared to $240 / \mathrm{mm}^{3} 1$ year earlier, with no leukocytosis. No abnormalities were noted on electrocardiogram, chest roentgenogram, or abdominal ultrasound.

EGD revealed diffuse white granular patches in the body and fundus of the stomach (shown in Fig. 1a-d). While the lesser curvature of the upper body appeared atrophic, regular arrangement of collecting venules was maintained. No abnormalities were noted in the antrum, esophagus, or duodenum. Biopsies of the white granular patches revealed infiltration of neutrophils, eosinophils, and lymphocytes in the interstitium. Of note, over 100 eosinophils per high-power field (hpf) were observed in all gastric biopsies (shown in Fig. 2a, b). Congo red stain was negative. Subsequent colonoscopy was unremarkable. Biopsies of the ileum showed normal lymphoid follicles and up to 22 eosinophils/hpf. Step biopsies of each colonic segment showed no notable eosinophilic infiltration.

Upon detailed questioning, the patient denied any abdominal or constitutional symptoms whatsoever. She also denied food or drug allergies, a family history of allergic disorders, ingestion of raw foods, overseas travel, insect bites, and sick contacts. Her bronchial asthma remained well controlled. Both the serum anti-Helicobacter pylori antibody titer and the urease breath test were negative. IgE was mildly elevated, to $260 \mathrm{IU} / \mathrm{mL}$. Rheumatoid factor as well as anti-nuclear, anti-Ro, anti-La, anti-neutrophil cytoplasmic, anti-aminoacyl tRNA synthetase, anti-intrinsic factor, and anti-parietal cell antibodies were all negative. Mild pollen allergies and no food allergies were noted in an extensive allergy panel. While the patient was still recommended to try a food elimination diet, she declined and was therefore observed without treatment.

The patient remained asymptomatic when she presented for a follow-up 5 months later. Her white blood cell count was $5,600 / \mathrm{mm}^{3}$, of which 460 were eosinophils. On follow-up EGD, the white granular patches persisted but had decreased in number and severity. However, gastric biopsies revealed eosinophilic infiltration of over 200/hpf, including a biopsy from the normal-appearing mucosa in the lower body of the stomach. Random biopsies of the duodenum showed no notable eosinophilic infiltration $(21 / \mathrm{hpf})$. The patient remained asymptomatic during another 6 months of follow-up.

\section{Discussion/Conclusion}

Unlike eosinophilic esophagitis, there are no established diagnostic criteria or clinical guidelines on EG [4, 5]. EG has classically been diagnosed based on 3 criteria: presence of gastrointestinal (GI) symptoms, histological evidence of eosinophilic infiltration in 1 or more segments of the GI tract, and the exclusion of other causes [6]. While GI symptoms in EG are often mild and nonspecific, the absence of any symptoms precludes the diagnosis [6, 7]. A peak concentration of at least 30 eosinophils/hpf in the gastric mucosa is required for the diagnosis of mucosal EG in most studies [3, 8]. Differential diagnoses of mucosal eosinophilia

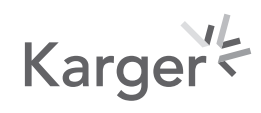



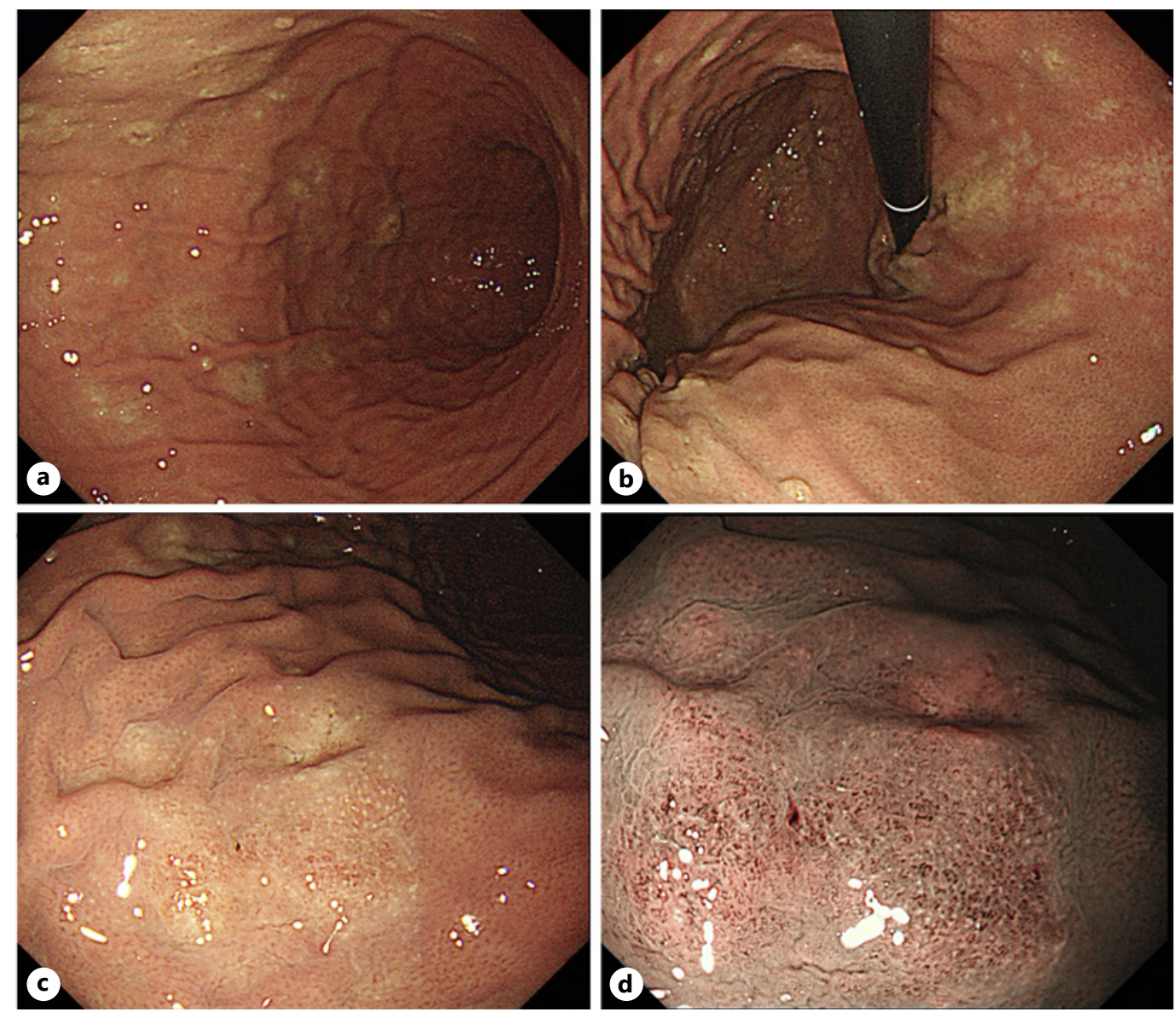

Fig. 1. Endoscopic findings. a Numerous white granular patches in the gastric body. $\mathbf{b}$ While the lesser curvature of the upper gastric body appeared atrophic, regular arrangement of collecting venules was maintained. c A closer view of a white, slightly elevated lesion with minimal signs of inflammation. d No surface or vascular irregularities suggestive of malignancy were observed on narrow-band imaging.

are broad and include Helicobacter pylori, cytomegalovirus, and parasitic infections, drug reactions, inflammatory bowel disease, hypereosinophilic syndrome, vasculitis, and connective tissue disease [8-10]. Causative autoimmune connective tissue disorders include systemic lupus erythematosus, rheumatoid arthritis, systemic sclerosis, and inflammatory myositis [9].

Endoscopic findings in EG are normal in a majority of cases, with various possible changes including erythema, erosions, fold thickening, nodules, and friability [10]. The white granular patches observed in our case are rare in EG but have been reported [10,11]. Patchy discoloration, which appeared similar to atrophic gastritis in our case, has also been reported [11].

Mild eosinophilia can be explained by various other causes, including asthma in our patient's case. Eosinophils are found in the normal gastric and intestinal mucosa, although they are not observed in the esophageal squamous epithelium. As such, mild eosinophilic infiltration can exist without causing symptoms. In a study of IgE-mediated peanut allergy patients undergoing oral immunotherapy, 40-60 eosinophils/hpf were found in stomach biopsies of 6 asymptomatic patients [12]. However, we found no asymptomatic reports of extreme eosinophilic infiltration of the gastric mucosa with endoscopic and histological findings consistent with EG. 

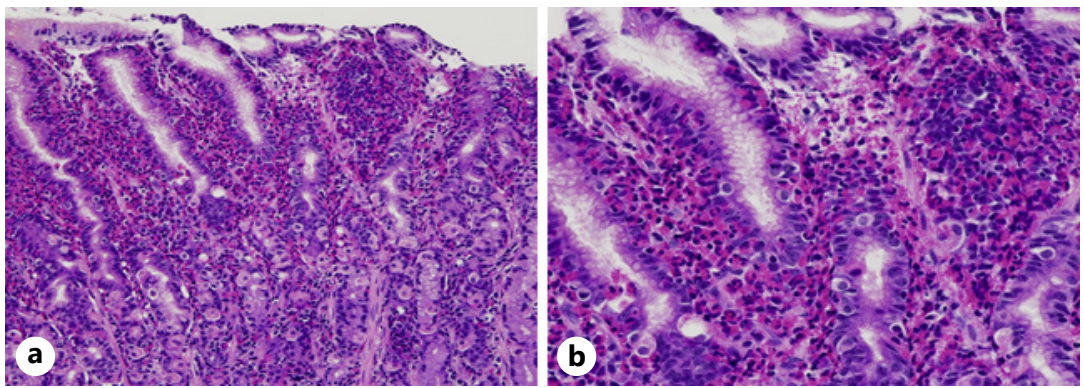

Fig. 2. Histological findings. Low-power (a) and high-power (b) magnification of a biopsy of a white granular patch revealed over 100 eosinophils per hpf, with particularly strong infiltration surrounding glands. hpf, high-power field.

There are no consensus statements or guidelines on the optimal treatment strategy for EG. Elemental diets or diets which eliminate common food allergens may be attempted, although reduced quality of life and adherence issues present significant hurdles in clinical practice [13]. Corticosteroids are generally the first choice in medical management, despite possible relapses when tapered or discontinued. Other antiallergic medications such as mast cell inhibitors and leukotriene receptor antagonists have been reported, but sufficient evidence supporting their use is lacking. Surgery is reserved for severe cases with obstructive symptoms.

On the other hand, spontaneous remission is not uncommon $[7,14]$. One study with a long-term follow-up found that no relapse was observed in $42 \%$ of patients, while $37 \%$ had relapsing and remitting disease and $21 \%$ developed chronic symptoms [14]. Another study found that most patients had improved clinical, endoscopic, and histological findings regardless of treatment [3]. While the study found correlations in clinical, endoscopic, and histological findings, cases like ours suggest that this may not always be the case.

In conclusion, we report a case of asymptomatic eosinophilic infiltration of the stomach accompanied by endoscopic and histological findings consistent with EG. As the clinical course of such patients remains unclear, close follow-up may be warranted until further evidence is accumulated.

\section{Acknowledgment}

This manuscript does not include any nonauthor contributors to acknowledge.

\section{Statement of Ethics}

The patient has given her written informed consent to publish this case (including publication of images). The identity of the patient has been protected. This case report complies with the guidelines for human studies and was conducted ethically in accordance with the World Medical Association Declaration of Helsinki. This study protocol was reviewed, and the need for approval was waived by the Ethics Committee at St. Luke's International Hospital.

\section{Conflict of Interest Statement}

The authors have no conflicts of interest to declare.

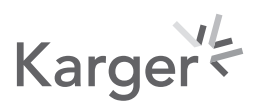


Yamamoto et al.: Asymptomatic Eosinophilic Gastritis

\section{Funding Sources}

This manuscript did not receive any funding.

\section{Author Contributions}

Kazuki Yamamoto and Takeshi Okamoto wrote the manuscript. Katsuyuki Fukuda critically reviewed the manuscript. All authors approved the final version of the manuscript.

\section{References}

1 Spergel JM, Book WM, Mays E, Song L, Shah SS, Talley NJ, et al. Variation in prevalence, diagnostic criteria, and initial management options for eosinophilic gastrointestinal diseases in the United States. J Pediatr Gastroenterol Nutr. 2011 Mar;52(3):300-6.

2 Kinoshita Y, Furuta K, Ishimaura N, Ishihara S, Sato S, Maruyama R, et al. Clinical characteristics of Japanese patients with eosinophilic esophagitis and eosinophilic gastroenteritis. J Gastroenterol. 2013 Mar;48(3): 333-9.

3 Pesek RD, Reed CC, Collins MH, Muir AB, Fulkerson PC, Menard-Katcher C, et al. Association between endoscopic and histologic findings in a multicenter retrospective cohort of patients with non-esophageal eosinophilic gastrointestinal disorders. Dig Dis Sci. 2020 Jul;65(7):2024-35.

4 Dellon ES, Liacouras CA, Molina-Infante J, Furuta GT, Spergel JM, Zevit N, et al. Updated international consensus diagnostic criteria for eosinophilic esophagitis: proceedings of the AGREE conference. Gastroenterology. 2018 Oct;155(4):1022-33.e10.

5 Dellon ES, Gonsalves N, Hirano I, Furuta GT, Liacouras CA, Katzka DA, et al. ACG clinical guideline: evidenced based approach to the diagnosis and management of esophageal eosinophilia and eosinophilic esophagitis (EoE). Am J Gastroenterol. 2013 May;108(5):679-93; quiz 693.

6 Talley NJ, Shorter RG, Phillips SF, Zinsmeister AR. Eosinophilic gastroenteritis: a clinicopathological study of patients with disease of the mucosa, muscle layer, and subserosal tissues. Gut. 1990 Jan;31(1):54-8.

7 Walker MM, Potter M, Talley NJ. Eosinophilic gastroenteritis and other eosinophilic gut diseases distal to the oesophagus. Lancet Gastroenterol Hepatol. 2018 Apr;3(4):271-80.

8 Yantiss RK. Eosinophils in the GI tract: how many is too many and what do they mean? Mod Pathol. 2015 Jan; 28(Suppl 1):S7-21.

9 Lecouffe-Desprets M, Groh M, Bour B, Le Jeunne C, Puéchal X. Eosinophilic gastrointestinal disorders associated with autoimmune connective tissue disease. Joint Bone Spine. 2016 Oct;83(5):479-84.

10 Khan S, Orenstein SR. Eosinophilic gastroenteritis. Gastroenterol Clin North Am. 2008 Jun;37(2):333-48.v.

11 Fujiwara Y, Tanoue K, Higashimori A, Nishida Y, Maruyama M, Itani S, et al. Endoscopic findings of gastric lesions in patients with eosinophilic gastrointestinal disorders. Endosc Int Open. 2020 Dec;8(12):E1817-25.

12 Wright BL, Fernandez-Becker NQ, Kambham N, Purington N, Tupa D, Zhang W, et al. Baseline gastrointestinal eosinophilia is common in oral immunotherapy subjects with IgE-mediated peanut allergy. Front Immunol. 2018 Nov; 9:2624.

13 Zhang M, Li Y. Eosinophilic gastroenteritis: a state-of-the-art review. J Gastroenterol Hepatol. 2017 Jan;32(1): 64-72.

14 Pineton de Chambrun G, Dufour G, Tassy B, Rivière B, Bouta N, Bismuth M, et al. Diagnosis, natural history and treatment of eosinophilic enteritis: a review. Curr Gastroenterol Rep. 2018 Jul;20(8):37. 